\title{
Villin is a biomarker for reverse polarity in colorectal micropapillary carcinoma
}

\author{
LI ZHAO, SHAO-YAN LIU, YU-MENG LI and ZHONG-TANG XIONG \\ Department of Pathology, The Third Affiliated Hospital of Guangzhou Medical \\ University, Guangzhou, Guangdong 510150, P.R. China
}

Received March 13, 2020; Accepted October 19, 2020

DOI: $10.3892 / 01.2020 .12333$

\begin{abstract}
Colorectal cancer (CRC) is a common malignant tumor of digestive system. CRC with micropapillary pattern (MPP) is an aggressive variant of colorectal adenocarcinoma. The aim of the present study was to clarify the clinicopathological significance and the prognostic role of an immunohistochemical marker, MPP, in CRC. The association between MPP and clinicopathological characteristics and prognosis in 286 cases of CRC (286/453 cases had follow-up information) were analysed. Then, 81 tissues without MPP and 90 tissues with MPP were analysed by immunohistochemistry using antibodies against villin, E-cadherin and epithelial membrane antigen (EMA). Bioinformatics was used to evaluate the expression of these three indicators in CRC. The proportion of micropapillary carcinoma in the overall tumour was $\geq 5 \%$, and was observed in 90/453 cases $(19.8 \%$ ). The present data showed that CRC with MPP displayed higher rates of vascular and lymphatic invasion, a higher metastatic lymph node ratio and a higher pathological tumour and metastasis stage compared with CRC without MPP. The positive expression rates of EMA, E-cadherin and villin were 50.3, 93.4 and $96.5 \%$, respectively. In 90 CRC cases with MPP, EMA inside-out pattern (I/OP) staining was observed in 26 cases $(28.9 \%)$, and it was often focal and partial, while 37 cases (41.1\%) had E-cadherin focal and partial staining compatible with reverse polarity. Villin I/OP staining was observed in 77 cases $(85.6 \%)$, and circumferential staining predominated over partial staining. Overall, the data suggested that the
\end{abstract}

Correspondence to: Dr Zhong-Tang Xiong, Department of Pathology, The Third Affiliated Hospital of Guangzhou Medical University, 63 Duobao Road, Guangzhou, Guangdong 510150, P.R. China

E-mail: zhongtang2000@126.com

Abbreviations: CRC, colorectal cancer; PDCs, poorly differentiated clusters; MPC, micropapillary carcinoma; I/OP, inside-out pattern; MPP, micropapillary pattern; EMA, epithelial membrane antigen; IHC, immunohistochemistry

Key words: colorectal carcinoma, micropapillary pattern, villin, EMA, E-cadherin presence of MPP is significantly associated with aggressive tumour behaviour and worse overall survival rate in CRC. Visualization and distinction of reverse polarity of colorectal micropapillary carcinomas is improved villin compared with EMA or E-cadherin.

\section{Introduction}

Colorectal carcinoma (CRC) is the second most commonly diagnosed cancer in women and the third in men around the world (1). Although the initial events of CRC development are relatively well understood and therapeutic approaches for early-stage disease have significantly improved (2), some aggressive types of CRC have not received enough attention from pathologists. These types of CRC may have higher rates of recurrence and metastasis, such as micropapillary cancer $(3,4)$. Therefore, it is important to identify specific markers to diagnose these forms of CRC.

Colorectal micropapillary carcinoma (MPC) is related to a poor prognosis (5). The tumour is characterized histologically by small papillary clusters of cells lacking a central fibrous vascular core located in lacunar spaces, with eosinophilic cytoplasm and pleomorphic nuclei (6-11). This pattern was described first in the breast (12) and ovarian cancer (13) and subsequently in other locations, including the urothelial (14), gastrointestinal (15), lung (16) and salivary gland cancer (17). An increasing number of studies have reported that MPC is an aggressive variant of colorectal adenocarcinoma $(4,11,18,19)$. However, there are only a few preliminary studies with small samples of CRC with micropapillary pattern (MPP) and a few case reports of clinicopathological studies.

MPC cells display reverse polarity and with the characteristics of an inside-out (I/O) epithelial membrane antigen $(20,21)$. I/O pattern (I/OP) staining of EMA/mucin (MUC)1 has also been confirmed in CRC with micropapillary components $(4,20,22,23)$. However, some studies have demonstrated that the sensitivity of EMA/MUC-1 staining is not high, and $>50 \%$ of cases have no EMA staining (4,23-25). Like EMA/MUC-1, villin is a surface-related glycoprotein and is also expressed in the normal colorectal mucosa (26). Using bioinformatics methods, the expression of villin, E-cadherin and EMA in colorectal cancer and normal mucosa was analysed. Immunohistochemical staining was used to compare the expression of EMA, E-cadherin and villin in 
colorectal MPC and common adenocarcinoma, aiming to identify improved markers of MPC.

The present study collected 453 colorectal cancer tissue samples between 2013 and 2018. The structure of colorectal cancer tissue was observed using microscopy. In total, 90 cases were accompanied by MPP and 85 cases of CRC with MPP and 201 cases without MPP were analysed to compare their clinicopathological parameters and the patient outcomes.

\section{Materials and methods}

Patients. The records of 453 patients who had undergone surgical resection of CRC at The Third Affiliated Hospital of Guangzhou Medical University (Guangzhou, China) between January 2013 and December 2018 were retrospectively analysed and followed up by telephone. The inclusion criteria were a complete follow-up, ranging from 1 to 58 months with a mean of 26 months. The patients consisted of 162 men and 124 women. The age of patients ranged from 27 to 93 years with a mean age of 60 years. The patient had not received any pre-operative chemotherapy or radiotherapy.

Clinicopathological characteristics, such as tumour size, differentiation, vascular and lymphatic invasion, lymph node metastasis and distant metastasis and pathological Tumour-Node-Metastasis (pTNM) stages (27), were reviewed using medical charts, pathological records and archived slides of tissue samples. All patients provided written informed consent and the study was approved by The Ethics Committee of The Third Affiliated Hospital (Guangzhou, China).

Evaluation of MPP. To elucidate the clinicopathological significance of MPCs, the presence of MPP was investigated in 453 CRCs by three pathologists in the present group. MPP is characterized by: i) A tumour cell nest, consisting of 3-20 tumour cells, with reverse polarity with an outer common border (6); ii) tumour cell clusters without fibrovascular cores (7); iii) tumour cells with pleomorphic nuclei and eosinophilic cytoplasm (8); and iv) a clear lacunar space around the tumour nest (11). In some cases, MPP in the mucin pools was determined. Comparisons between CRCs with MPP and without MPP were conducted.

GEPIA and the Human Protein Atlas (HPA) database analysis. Villin, EMA and E-cadherin expression in colorectal adenocarcinoma (COAD) and normal tissues were obtained from the GEPIA database (http://gepia.cancer-pku.cn/). Immunohistochemistry results of COAD with three different antibodies against villin, five different antibodies against EMA/MUC-1 and E-cadherin, were obtained from the HPA database (https://www.proteinatlas.org/), which has been generated from RNA-sequencing analysis and immunohistochemistry analysis (28). The HPA database analyzes the expression of villin, E-cadherin and EMA/MUC-1 antibodies in colorectal cancer to compare the differences between different clone antibodies, which is helpful for us to select more effective antibodies.

Tissue microarray construction. The hematoxylin and eosin (H\&E)-stained slides and corresponding paraffin-embedded tissue (29) blocks of COAD with MPP were obtained from
Table I. Percentage of micropapillary pattern within the 90 cases.

\begin{tabular}{lc} 
Percentage, $\%$ & Number o \\
\hline 5 & 11 \\
10 & 37 \\
15 & 10 \\
20 & 11 \\
25 & 7 \\
30 & 5 \\
40 & 5 \\
50 & 3 \\
60 & 4 \\
70 & 1 \\
\hline
\end{tabular}

the Department of Pathology (The Third Affiliated Hospital of Guangzhou Medical University). Representative areas of MPC and conventional CRC were marked with a marker pen on H\&E slides. Then these corresponding areas of paraffin-embedded tissue blocks were marked by the same method. Tissue microarrays (TAMs) were constructed using a tissue-arraying instrument (QuickRay; Unitma Co., Ltd.). Briefly, tissue cores with a $1.5-\mathrm{mm}$ diameter of MPC and conventional CRC were obtained from donor tissue blocks, and were transferred to two recipient paraffining blocks, respectively. The tissues used for the TAMs were the same as those aforementioned.

Immunohistochemistry. Consecutive 4- $\mu \mathrm{m}$ thick unstained sections were cut from TAM blocks for immunohistochemical staining, which was performed using the Leica automatic immunostaining device (Leica Microsystems, Inc.). Primary antibodies against villin (1:100; cat. no. PA0554, Leica Microsystems, Inc.), E-cadherin (1:100; cat. no. GT210701) and EMA (1:100; cat. no. GM061301) (both Gene Tech Biotechnology Co., Ltd.). The immunohistochemistry profile included the expression of villin, E-cadherin and EMA.

Immunohistochemistry were conducted according to previously described methods (24). All slides were reviewed and scored independently by three pathologists. The pathologists were blinded to the experiment. The scoring method based on both the intensity ( 0 , no staining; 1 , weak staining; 2 , medium staining; 3 , strong staining) and proportion of positive cells $(0,0 \% ; 1,1-25 \% ; 2,26-75 \%$; $3,76-100 \%)$. The final staining score was calculated by multiplying the staining intensity score by extent of staining score. A final staining score of $\geq 3$ was considered positive, and others were classified as low expression. The cases of positive expression were further classified into two groups based on the location of the three markers. For MUC-1 and villin, these cases with positive expression on the outer borders of tumor cell clusters were regarded as having reversion of cell polarity (I/OP staining group), and staining in the apical membrane of adenocarcinoma were regarded as non-reversion of cell polarity (no I/OP staining group). For E-cadherin, the cases with cup-shaped expression patterns around cell nests were 

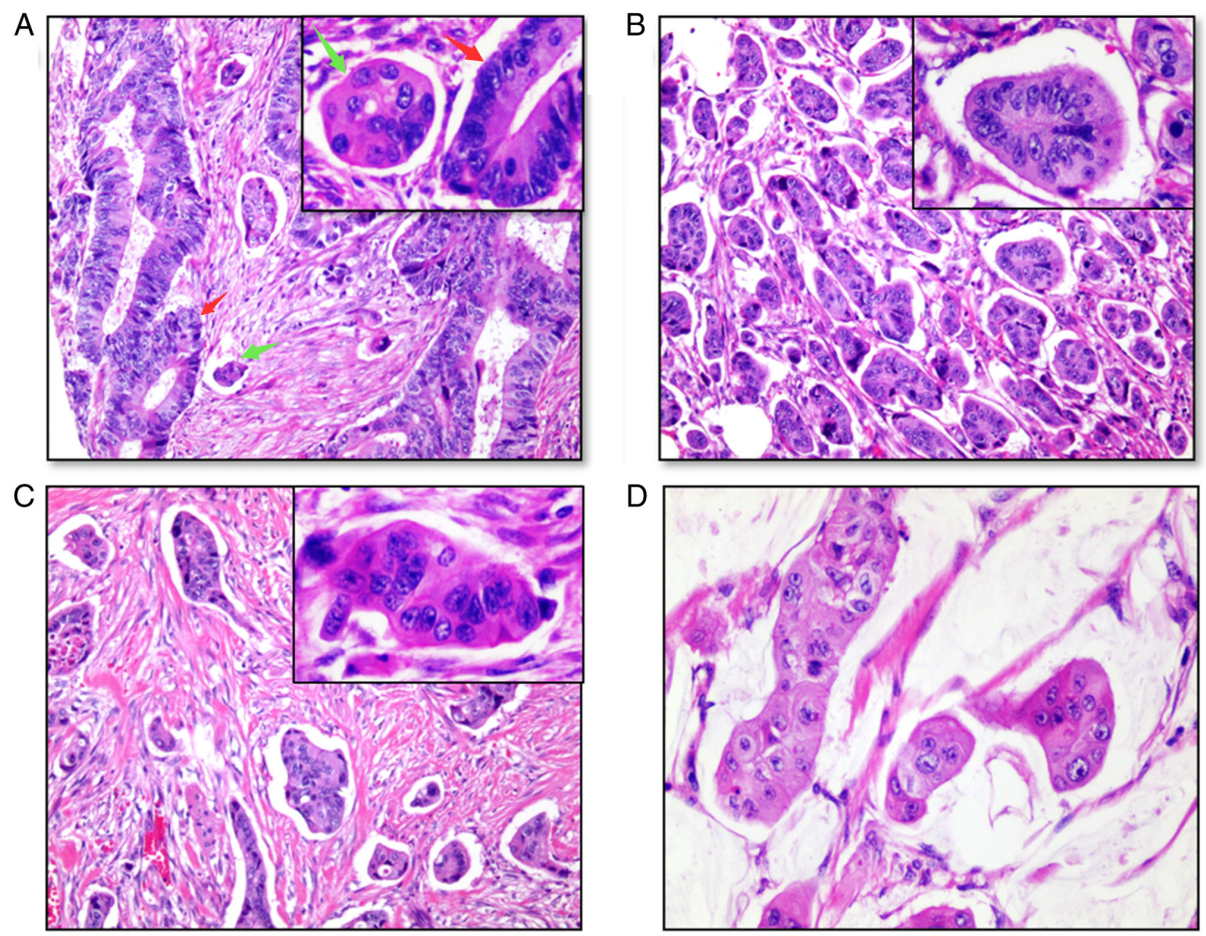

Figure 1. Representative images showing features of MPP in CRCs (magnification, x100). (A) Mixed adenocarcinoma of CRC containing moderately differentiated tubular adenocarcinoma and micropapillary carcinoma. Red arrow shows conventional tubular adenocarcinoma and the green arrow shows micropapillary carcinoma. (B) Adenocarcinomas with MPP are usually small, have no fibrovascular cores and have cells with pleomorphic nuclei and eosinophilic cytoplasm. (C) Cleft-like spaces between tumour clusters and stroma. (D) Adenocarcinoma with a MPP in mucin pools. MPP, micropapillary pattern; CRC, colorectal carcinoma.

regarded as having reversion of cell polarity (I/OP staining group) and complete cell membrane expression were regarded as non-reversion of cell polarity (no I/OP staining).

Statistical analysis. Statistical analyses were performed using SPSS version 19.0 software (IMB Corp.). Data are presented as value (\%), unless otherwise shown. The significance of the association between the presence of MPP and the clinicopathological characteristics was determined by either the $\chi^{2}$ test or Fisher's exact test (two-sided). Survival curves were estimated using the Kaplan-Meier product-limit method, and differences between the survival curves were determined using the log-rank test. The statistical method used in the GEPIA database was one-way ANOVA with Least Significance Difference post hoc test. $\mathrm{P}<0.05$ was considered to indicate a statistically significant difference.

\section{Results}

Histological features of primary COAD with MPP. A MPP with a proportion $\geq 5 \%$ of the tumour was identified in 90/453 cases (19.87\%) of CRC. The percentage of MPP within the 90 cases ranged from $5-70 \%$, as summarized in Table I. The morphology of MPP was different from that of conventional adenocarcinoma (Fig. 1A). The glandular tube structure can be seen in most conventional adenocarcinomas, while the MPP shows nest-mass transformation. MPP was often present at the invasive edge of the tumour. The main feature of the MPP was the presence of clefts between the stroma, devoid of central fibrovascular cores and with pleomorphic nuclei and eosinophilic cytoplasm (Fig. 1B). There were tumour cell nests of MPP, consisting of 3-20 tumour cells with reverse polarity and with an outer common border (Fig. 1C). In some cases, adenocarcinoma with MPP in mucin pools was observed (Fig. 1D). These were the histological features of MPP.

COAD with the MPP predicts a poor outcome and promotes disease progression. Follow-up data was collected for 286 patients. Their age ranged from 27 to 93 years (mean 60 years), and 162 patients were men and 124 patients were women. The tumour size ranged from 0.3 to $16.0 \mathrm{~cm}$ (mean $4.7 \mathrm{~cm}$ ). The mean number of total lymph nodes dissected was 11.4 (range, 0-29), and the mean number of positive lymph nodes was 1.5 (range, 1-14). Of 286 cases, 201 carcinomas were COAD, not otherwise specified, while 85 were MPC (all data not shown).

A comparison of the clinicopathological features of CRC with and without MPP is summarized in Table II. There was no significant difference between age, sex, tumour size and tumour differentiation. Meanwhile, carcinomas with MPP were significantly associated with a more advanced $\mathrm{T}(\mathrm{P}<0.001$ T4 vs. T1-2), $\mathrm{N}(\mathrm{P}<0.001 \mathrm{~N} 0$ vs. N1-N2) and $\mathrm{M}$ stage $(\mathrm{P}=0.024)$, and higher levels of lymphovascular invasion $(\mathrm{P}=0.009)$ and higher TNM stages $(\mathrm{P}<0.001 \mathrm{I}-\mathrm{II}$ vs. IV). Consistent with these results, Kaplan-Meier analysis also showed a positive association between tumours with MPP and worse overall survival rate ( $\mathrm{P}=0.005$; Fig. 2). Kaplan Meier analysis showed no significant association between depth of invasion ( $\mathrm{T}$ stage) and overall survival (Fig. S1A). Tumor size was also not significantly associated with worse overall survival (Fig. S1B). 
Table II. Comparison of clinicopathological data between 85 cases of MPC and 201 cases of non-MPC.

\begin{tabular}{|c|c|c|c|c|c|}
\hline $\begin{array}{l}\text { Clinicopathological } \\
\text { variables }\end{array}$ & Value, $\mathrm{n}$ & $\begin{array}{l}\text { MPC, } \\
\mathrm{n}(\%)\end{array}$ & $\begin{array}{l}\text { Non-MPC, } \\
\mathrm{n}(\%)\end{array}$ & $\chi^{2}$ & P-value \\
\hline Age, years & & & & 0.469 & 0.577 \\
\hline$<60$ & 88 & $23(27.1)$ & $65(32.3)$ & & \\
\hline$\geq 60$ & 198 & $62(72.9)$ & $136(67.7)$ & & \\
\hline Sex & & & & 0.807 & 0.434 \\
\hline Male & 162 & $53(60.2)$ & $109(54.2)$ & & \\
\hline Female & 124 & $32(39.8)$ & $92(45.8)$ & & \\
\hline Tumor size, $\mathrm{cm}$ & & & & 0.318 & 0.605 \\
\hline$<5$ & 149 & $43(50.6)$ & $109(54.2)$ & & \\
\hline$\geq 5$ & 137 & $42(49.4)$ & $92(45.8)$ & & \\
\hline Tumor differentiation & & & & 0.298 & 0.862 \\
\hline Well & 59 & $17(20.0)$ & $42(20.9)$ & & \\
\hline Moderate & 217 & $66(76.5)$ & $152(75.6)$ & & \\
\hline Poor & 10 & $2(3.5)$ & $7(3.5)$ & & \\
\hline T classification & & & & 4.942 & 0.031 \\
\hline $\mathrm{T} 1-\mathrm{T} 2$ & 36 & $5(5.9)$ & $31(15.4)$ & & \\
\hline T3-T4 & 250 & $80(94.1)$ & $170(84.6)$ & & \\
\hline $\mathrm{N}$ classification & & & & 13.455 & $<0.0001$ \\
\hline No & 116 & $20(23.5)$ & $94(46.8)$ & & \\
\hline N1-N2 & 170 & $65(76.5)$ & $107(53.2)$ & & \\
\hline M classification & & & & 5.07 & 0.024 \\
\hline M0 & 241 & $66(77.6)$ & $177(88.1)$ & & \\
\hline M1 & 45 & $21(22.4)$ & $24(11.9)$ & & \\
\hline Lymphovascular invasion & & & & 7.089 & 0.009 \\
\hline No invasion & 93 & $18(21.2)$ & $75(37.5)$ & & \\
\hline Invasion & 193 & $67(78.8)$ & $126(62.5)$ & & \\
\hline Stage & & & & 15.510 & $<0.0001$ \\
\hline I-II & 103 & $16(18.8)$ & $87(43.3)$ & & \\
\hline III-IV & 183 & $69(81.2)$ & $114(56.7)$ & & \\
\hline
\end{tabular}

MPC, micropapillary carcinoma; $\mathrm{T}$, tumour; N, node; $\mathrm{M}$, metastasis.

Although, Cox regression analyses showed that CRC with MPP was not an independent prognostic factor for the outcomes of patients, age was an independent prognostic factor (Table III).

Bioinformatics analysis of villin expression in $C R C$. I/OP staining of EMA/MUC-1 and E-cadherin has been recognized as two hallmarks of MPCs (20-22), especially EMA/MUC-1 (23,25). Villin is another surface-related glycoprotein similar to EMA/MUC-1, which is also present in the normal colonic mucosa (26).

To clarify the significance of these three surface-related glycoproteins in CRC, the expression differences in CRC tissues and normal tissues were analysed using bioinformatics. Based on the GEPIA database, as shown in Fig. 3A, there was no difference between COAD tissues and normal tissues for the transcription level of EMA/MUC-1. The mRNA expression of E-cadherin and villin was higher in COAD tissues compared with in normal tissues (Fig. 3B and C, respectively).

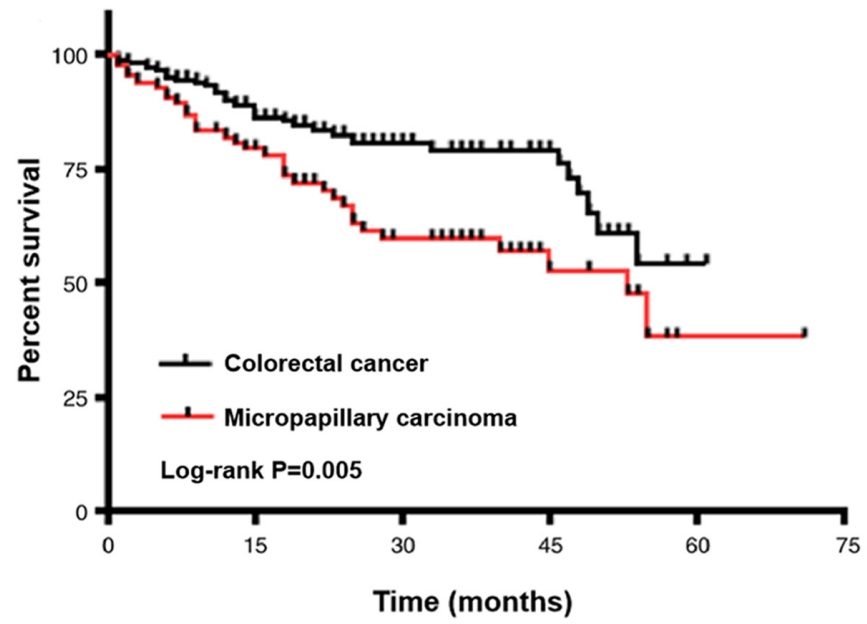

Figure 2. MPP predicts poor outcome in patients with CRC. Kaplan-Meier analysis of overall survival rate in all patients with CRC according to MPP. Data are presented as means \pm SD from three independent experiments. 
Table III. Summary of overall survival rate analysis by univariate and multivariate Cox regression analysis.

\begin{tabular}{|c|c|c|c|c|c|c|}
\hline \multirow[b]{2}{*}{ Variables } & \multicolumn{3}{|c|}{ Univariate analysis } & \multicolumn{3}{|c|}{ Multivariate analysis } \\
\hline & P-value & HR & $95 \% \mathrm{CI}$ & P-value & HR & $95 \% \mathrm{CI}$ \\
\hline Age, $<60$ vs. $\geq 60$ years & 0.004 & 2.479 & $1.336-4.602$ & 0.007 & 2.341 & $1.258-4.358$ \\
\hline T classification, T1-T2 vs. T3-T4 & $<0.001$ & 2.144 & $1.458-3.154$ & & & \\
\hline $\mathrm{N}$ classification, N0 vs. N1-N2 & $<0.001$ & 3.535 & $1.975-6.329$ & & & \\
\hline M classification, M0 vs. M1 & $<0.001$ & 2.833 & $1.684-4.766$ & & & \\
\hline Lymphovascular invasion, yes vs. no & 0.002 & 2.361 & $1.358-4.104$ & & & \\
\hline Stage, I-II vs. III-IV & $<0.001$ & 2.303 & $1.693-3.235$ & $<0.001$ & 2.314 & $1.663-3.222$ \\
\hline MPC vs. non-MPC & 0.008 & 1.855 & $1.174-2.933$ & & & \\
\hline
\end{tabular}

HR, hazard ratio; CI, confidence interval; MPC, micropapillary carcinoma; T, tumour; N, node; M, metastasis.

A

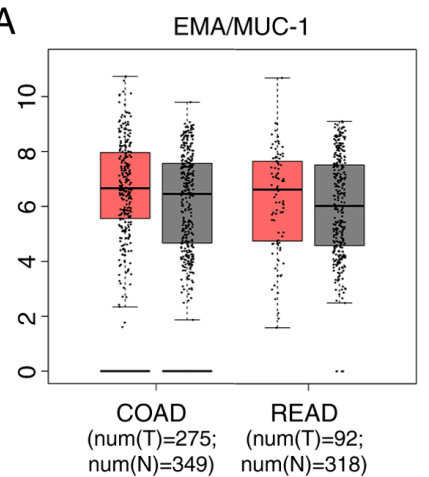

$\mathrm{D}$

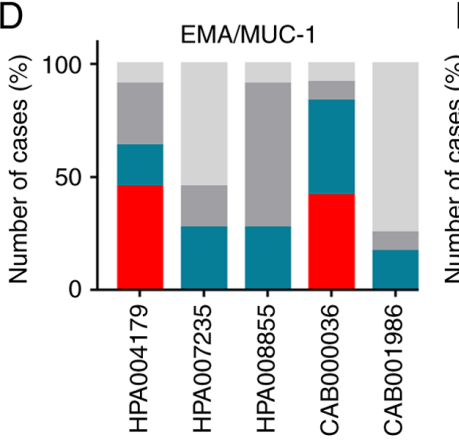

G

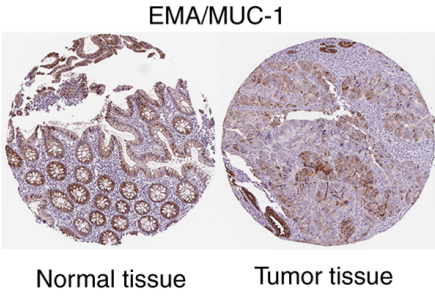

B

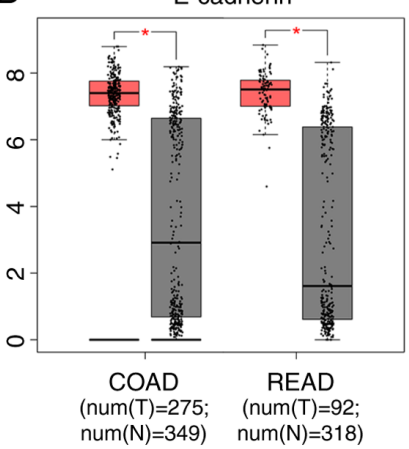

$\mathrm{E}$

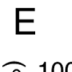

E-cadherin

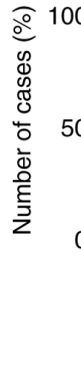

$\mathrm{H}$

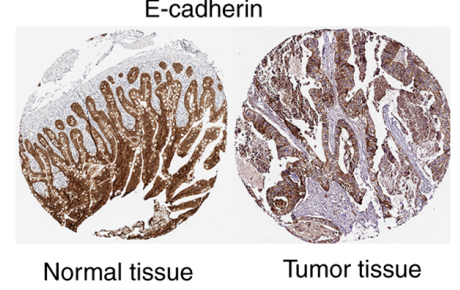

$\mathrm{F}$
C
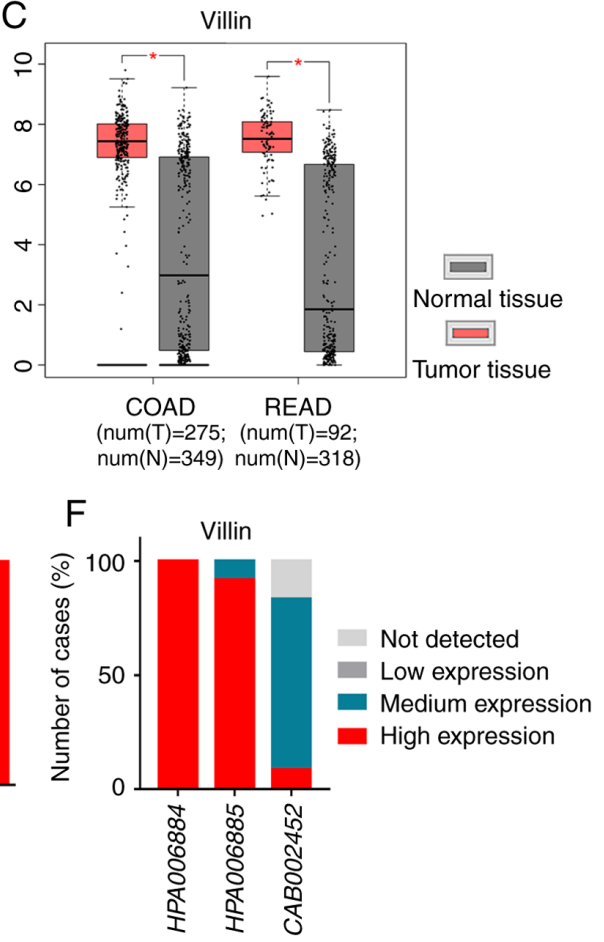

I

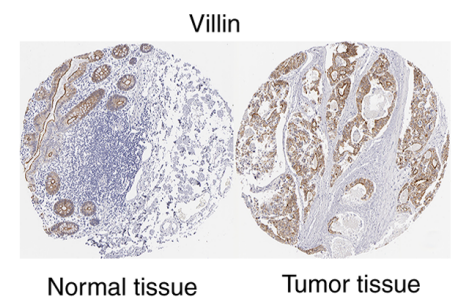

Figure 3. Expression level of EMA/MUC-1, E-cadherin and villin in colorectal cancer tissue samples. (A) EMA expression was not significantly different between carcinoma and non-cancerous samples. mRNA expression level of (B) E-cadherin (C) villin were significantly higher in COAS and READ compared with in normal tissues. Different antibodies of (D) EMA/MUC-1, (E) E-cadherin and (F) villin immunohistochemical results from patients with COAD using the HPA database. Immunohistochemical expression patterns of $(\mathrm{G})$ EMA/MUC-1, $(\mathrm{H})$ E-cadherin and (I) villin in conventional CRC and normal intestinal mucosa from the HPA database. ${ }^{*} \mathrm{P}<0.05$ vs. non-cancerous tissue. EMA/MUC-1, epithelial membrane antigen/mucin 1; COAD, colorectal adenocarcinoma; READ, rectum adenocarcinoma; T, tumour; N, normal; HPA, Human Protein Atlas.

The HPA database is a repository of transcriptomics and proteomics data generated from RNA sequencing and immunohistochemistry analysis (28). The present study analysed
EMA/MUC-1, E-cadherin and villin immunohistochemical results from patients with COAD using the HPA database. In total, five different EMA/MUC-1 antibodies were analysed 

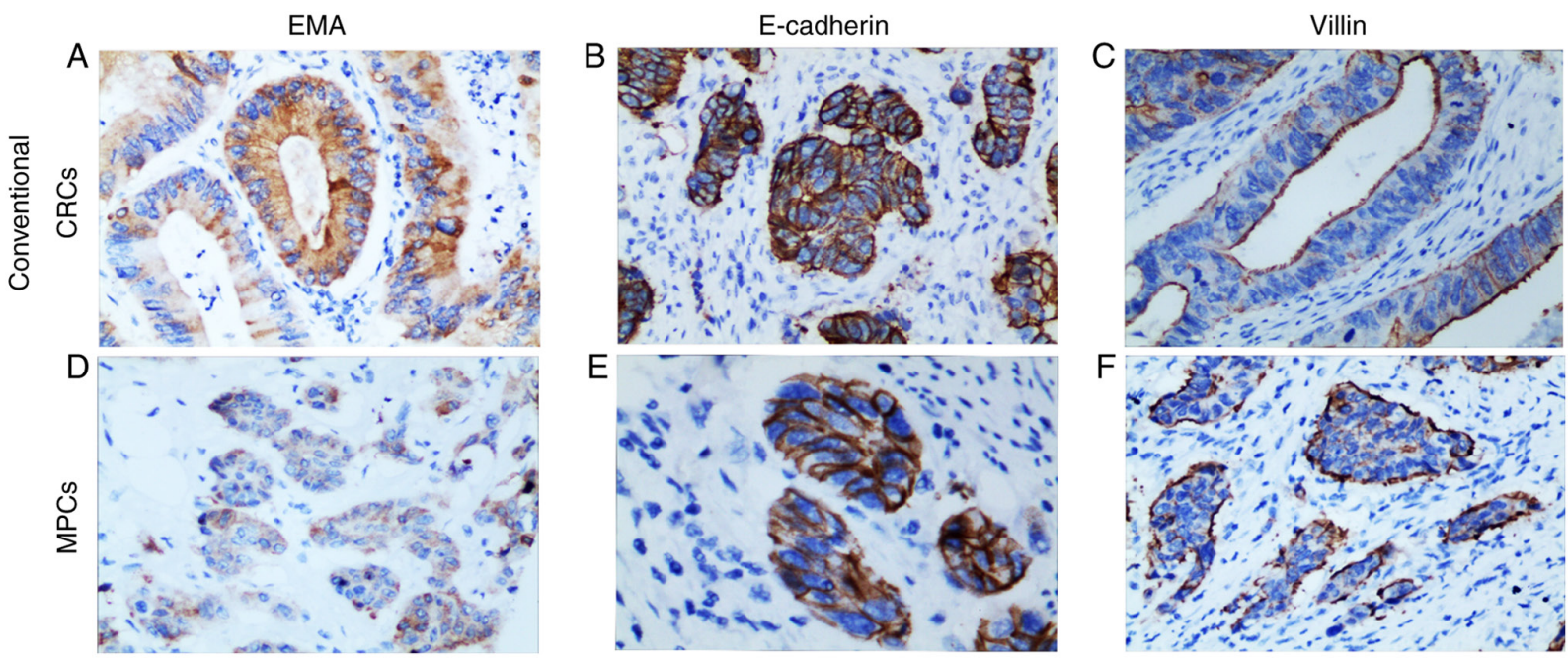

Figure 4. Expression characteristics of EMA, E-cadherin and villin in MPC. (A) EMA, (B) E-cadherin and (C) villin are expressed in conventional CRC (magnification, x200). (D) EMA and (E) E-cadherin partly show the inside-out pattern of neoplastic cells in MPC. (F) Villin evidently highlights the reverse polarity of the micropapillary pattern. EMA, epithelial membrane antigen; CRC, colorectal cancer; MPC, micropapillary carcinoma.

Table IV. Immunohistochemical results for 81 cases of nonMMP and 90 cases of MPP.

\begin{tabular}{lcc}
\hline Protein & Non-MPP, $\mathrm{n}(\%)$ & With MPP, n (\%) \\
\hline Villin & & \\
Y & $3(3.7)$ & $17(85.6)$ \\
N & $74(91.3)$ & $2(2.2)$ \\
U & $4(5.0)$ & \\
E-cadherin & & $37(41.1)$ \\
Y & $2(2.5)$ & $47(52.2)$ \\
N & $74(91.3)$ & $6(6.7)$ \\
U & $5(6.2)$ & \\
EMA & & $26(28.9)$ \\
Y & $1(1.2)$ & $17(18.9)$ \\
N & $42(51.9)$ & $47(52.2)$ \\
U & $38(46.9)$ & \\
\hline
\end{tabular}

MPP, micropapillary pattern; Y, detected with I/OP staining. N, not detected with I/OP staining; U, no staining; I/OP, inside-out pattern; EMA, epithelial membrane antigen.

and it was demonstrated that EMA/MUC-1 had the highest positive expression rate with $\mathrm{CAB} 000036$, with high expression accounting for $42 \%$, middle expression accounting for $42 \%$ and low expression accounting for $8 \%$. For CAB001986, the positive expression rate of EMA/MUC-1 was only 25\% (Fig. 3D). E-cadherin had high expression in $>99 \%$ of CRC cases (Fig. 3E). In total, three different villin antibodies were compared and the positive expression rates were 100, 100 and $83.3 \%$, and villin had high expression in all cases with HPA006884 (Fig. 3F). These results showed that villin and E-cadherin are more sensitive compared with EMA/MUC-1 in COAD.

In addition, the subcellular locations of EMA, E-cadherin and villin in CRCs and normal tissues were also investigated using the HPA database (30). The results demonstrated that MUC-1 protein was expressed in the cytoplasm and on the apical surface of glandular epithelial cells (Fig. 3G). E-cadherin was expressed on the cellular membrane, but absent expression on the apical surface of glandular cells, presenting as cup-like staining pattern (Fig. 3H). Similar to E-cadherin, villin had high expression on the apical surface of glandular cells and weak expression in the cytoplasm (Fig. 3I). Fig. S1 also demonstrates the IHC expression patterns of EMA, E-cadherin and villin in normal intestinal mucosa and conventional CRC, with similar results to Fig. 3G-I.

Immunohistochemical evaluation. Among the 90 conventional CRCs, nine cases were excluded because of the loss of tissue cores. Thus, 81 conventional CRCs and 90 MPC cases were successfully examined using IHC. The IHC results of EMA, E-cadherin and villin in the conventional CRCs and MPCs are summarized in Table IV. In the 81 conventional CRCs, EMA positive expression was detected in 43 (53.1\%), E-cadherin positive expression in $76(93.8 \%)$ and villin positive expression in 77 (95.0\%). Among the 90 MPC cases, EMA positive expression was detected in $43(47.8 \%)$, E-cadherin positive expression in $84(93.3 \%)$ and villin positive expression in 88 (97.8\%). The IHC expression results (Table IV) showed that villin expression was lost in six cases, all of which were poorly differentiated CRC. The rates of positive expression for EMA, E-cadherin and villin were not statistically different between conventional CRCs and MPCs. However, the rate of positive expression of EMA was $<60 \%$ in conventional CRCs and MPCs. EMA, E-cadherin and villin in non-MPP of CRCs generally displayed a similar profile of staining, namely luminal staining (Fig. 4A-C).

As shown in Table IV, among the cases with positive expression for EMA, reverse polarity of the tumour cells was detected in 26 (60.5\%) MPCs (Fig. 4D). E-cadherin was expressed on the cellular membrane. E-cadherin expression showed that 37 (44\%) MPCs had inverse tumour cell polarity (Fig. 4E). 
Among most of the conventional CRCs, villin was highly expressed on the apical surface of the glandular cells and weakly expressed of absent in the cytoplasm. In the MPCs, there was high villin expression on the outer borders of tumour cell clusters and weak or absent expression in the centre of the tumour cell clusters, displaying reversed cell polarity. According to villin expression, 77 (87.5\%) MPCs had inverse tumour cell polarity (Fig. 4F). The results of immunohistochemistry showed that compared with EMA and E-cadherin, villin had an improved ability to recognize MPP.

\section{Discussion}

Cancer with MPP shows distinct histology characterized by an eosinophilic cell cluster (6-8), a lack of a fibrous blood vessel axis $(9,10)$, voids around the cell mass and cell polarity reversal (31). Pure MPC is rare and has only been reported in the breast, pancreatic and colon cancer $(6,32)$. Previous research has reported that the incidence of CRC with MPP is $9.4-27.8 \%(6,33,34)$, and it was accompanied by aggressive histological features, including higher levels of lymphovascular and perineural invasion, more frequent lymph node and distant metastases and higher TNM stages $(6,18,34,35)$. The present study, consistent with previous studies $(3,4,6)$, identified $19.8 \%$ of CRCs with a MPC component (90/453 cases). It was also reported that $\mathrm{CRC}$ with MPP was significantly associated with more frequent lymph node metastasis and higher TNM stages.

MPP should be distinguished from poorly differentiated clusters (PDCs) and tumour budding (36). MPP generally consists of 3-20 cells with cleft-like spaces and without fibrovascular cores at the interface with the stroma (6-10). PDCs are composed of no less than five cancer cells, generally located on the invasive margin and without glandular formation (37). Tumour budding refers to single cells or small clusters of dedifferentiated tumour cells (38). The epithelial-mesenchymal transition can occur in aforementioned three pathological forms, leading to decreased expression of E-cadherin in epithelial cells $(37,38)$. The peripheral space may be an important diagnostic clue to distinguish MPP from PDCs. Hence, the morphological and immunohistochemical expressions of MPP, PDCs and tumour budding are similar, so they can be misdiagnosed. As CRC with MPP is more aggressive compared with non-MPP CRC, pathologists need to carefully look for MPC in colorectal biopsy materials $(27,34,39)$.

I/OP staining of EMA was first detected in breast cancer with micropapillary components and subsequently reported in micropapillary carcinomas of the colorectum (40). E-cadherin is a calcium-dependent cell-cell adhesion glycoprotein comprised of five extracellular cadherin repeats, encoded by the gene cadherin 1 (41). E-cadherin is a classical member of the cadherin superfamily of proteins and is often used as a useful antibody in the diagnosis of micropapillary cancer (27). However, the expression of this protein has not specifically analysed in colon cancer with MPP (35).

Villin is also a member of a family of calcium-regulated actin-binding proteins (26). This protein represents a dominant part of the brush border cytoskeleton that functions in the capping, severing and bundling of actin filaments (42). In normal intestinal epithelium and colorectal cancer, villin can be seen on the apical/luminal surface $(26,42)$. The present bioinformatics analysis demonstrated that EMA had the lowest sensitivity in COAD compared with villin and E-cadherin, as reported by previous studies $(20,22,23,25)$. A previous study reported that villin detection using IHC is useful in the detection of reverse polarity of MPP (19).

The current IHC results were consistent with those reported in the literature $(19,20,22)$, demonstrating that, when identifying the MPC of CRC, the sensitivity and specificity of villin is higher compared with that of E-cadherin and EMA. E-cadherin is one of the immunomarkers of $\operatorname{MPC}(27,37,38)$, but the current study and previous studies showed that it often had no staining or only partial staining (35). In the present results, the I/OP staining of EMA and E-cadherin showed poor sensitivity and was often focal rather than the diffuse I/OP staining usually shown in illustrations $(20,23)$. However, villin expression was high on the outer borders of tumour cell clusters and was weakly expressed or absent in the centre of the tumour cell clusters, displaying reversed cell polarity. The present study demonstrated that villin was a useful marker of colorectal MPC and had greater sensitivity for the detection of reverse polarity compared with EMA and E-cadherin. Overall, the current data may help improve the clinical diagnosis of MPC of the colorectum.

There are limitations to the present study. Western blotting was not used to confirm the differential expression of EMA, E-cadherin and villin in normal mucosa or in conventional CRC and colorectal MPC. In addition, the molecular mechanisms underlying the carcinogenesis and tumour progression of this unique morphological pattern need to be elucidated in future research. For example, second generation sequencing could be used to find differentially expressed genes, screen regulatory molecules, find relevant signalling pathways and clarify the relationship between upstream and downstream regulation.

In summary, an MPP is not an infrequent finding in CRC. CRC with a micropapillary component is significantly associated with lymphovascular invasion and a higher TNM stage. MPC is a histological prognostic factor of poor survival. Accurate identification of micropapillary components is essential to determine patient prognosis and to improve clinical management. The I/OP staining with EMA and E-cadherin ranged from diffuse circumferential through focal and partial to absent, and in most cases, villin showed this pattern of staining more clearly compared with EMA and E-cadherin.

\section{Acknowledgements}

Not applicable.

\section{Funding}

No funding was received.

\section{Availability of data and materials}

All data generated or analyzed during this study are included in this published article. Additional datasets generated and/or analyzed during the current study are available in the GEPIA (http://gepia.cancer-pku.cn/) repository and the Human Protein Atlas (https://www.proteinatlas.org/) repository. 


\section{Authors' contributions}

LZ, SYL, YML contributed to the reviewing the literature, integrating and analyzing the literature results and the collection of patient data. LZ, YML and ZTX designed the study and analyzed the data. SYL performed the immunohistochemistry, and LZ, ZTX and YML participated in the immunohistochemical scoring. LZ contributed to the statistical analysis. ZTX supervised all the work. All authors read and approved the final manuscript.

\section{Ethics approval and consent to participate}

The study was approved by The Ethics Committee of The Third Affiliated Hospital (Guangzhou, China) and all patients provided informed written consent.

\section{Patient consent for publication}

Not applicable.

\section{Competing interests}

The authors declare that they have no competing interests.

\section{References}

1. Torre LA, Bray F, Siegel RL, Ferlay J, Lortet-Tieulent J and Jemal A: Global cancer statistics, 2012. CA Cancer J Clin 65 87-108, 2015.

2. Tauriello DV, Calon A, Lonardo E and Batlle E: Determinants of metastatic competency in colorectal cancer. Mol Oncol 11: 97-119, 2017.

3. Remo A, Fassan M, Vanoli A, Bonetti LR, Barresi V, Tatangelo F, Gafà R, Giordano G, Pancione M, Grillo F, et al: Morphology and molecular features of rare colorectal carcinoma histotypes. Cancers (Basel) 11: E1036, 2019.

4. Guo Z, Yang Z, Li D, Tang J, Xu J, Shen H and Yuan Y: Colorectal cancer with invasive micropapillary components (IMPCs) shows high lymph node metastasis and a poor prognosis: A retrospective clinical study. Medicine (Baltimore) 99: e20238, 2020.

5. Muratsu A, Noura S, Matsumura T, Hirota M, Yasuyama $\mathrm{H}$, Koga C, Takata A, Kameda C, Murakami M, Kawabata R, et al: A colorectal cancer with invasive micropapillary carcinoma. Gan To Kagaku Ryoho 44: 1814-1816, 2017 (In Japanese)

6. Haupt B, Ro JY, Schwartz MR and Shen SS: Colorectal adenocarcinoma with micropapillary pattern and its association with lymph node metastasis. Mod Pathol 20: 729-733, 2007.

7. Patankar M, Väyrynen S, Tuomisto A, Mäkinen M, Eskelinen S and Karttunen TJ: Micropapillary structures in colorectal cancer: An anoikis-resistant subpopulation. Anticancer Res 38: 2915-2921, 2018.

8. Fujita T, Konishi M, Gotohda N, Takahashi S, Nakagohri T, Kojima M and Kinoshita T: Invasive micropapillary carcinoma of the ampulla of Vater with extensive lymph node metastasis: Report of a case. Surg Today 40: 1197-1200, 2010.

9. Vardar E, Yardim BG, Vardar R and Ölmez M: Primary gastric invasive micropapillary carcinoma: A case report. Turk Patoloji Derg 31: 219-222, 2015.

10. Ushiku T, Matsusaka K, Iwasaki Y, Tateishi Y, Funata N, Seto $\mathrm{Y}$ and Fukayama M: Gastric carcinoma with invasive micropapillary pattern and its association with lymph node metastasis. Histopathology 59: 1081-1089, 2011.

11. Wen $\mathrm{P}, \mathrm{Xu} \mathrm{Y}$, Frankel WL and Shen R: Invasive micropapillary carcinoma of the sigmoid colon: Distinct morphology and aggressive behavior. Int J Clin Exp Pathol 1: 457-460, 2008.

12. Collins K and Ricci A Jr: Micropapillary variant of mucinous breast carcinoma: A distinct subtype. Breast J 24: 339-342, 2018.

13. Young RH: Ovarian tumors: A survey of selected advances of note during the life of this journal. Hum Pathol 95: 169-206, 2020 .
14. Shinagawa T, Hoshino H, Taga M, Sakai Y, Imamura Y, Yokoyama $\mathrm{O}$ and Kobayashi M: Clinicopathological implications to micropapillary bladder urothelial carcinoma of the presence of sialyl Lewis X-decorated mucin 1 in stroma-facing membranes. Urol Oncol 35: 606.e17-606.e23, 2017.

15. Guzińska-Ustymowicz K, Niewiarowska K and Pryczynicz A: Invasive micropapillary carcinoma: A distinct type of adenocarcinomas in the gastrointestinal tract. World J Gastroenterol 20: 4597-4606, 2014.

16. Monroig-Bosque PDC, Morales-Rosado JA, Roden AC, Churg A, Barrios R, Cagle P, Ge Y, Allen TC, Smith ML, Larsen BT, et al: Micropapillary adenocarcinoma of lung: Morphological criteria and diagnostic reproducibility among pulmonary pathologists. Ann Diagn Pathol 41: 43-50, 2019.

17. Otsuru M, Aoki T, Kondo Y, Ota Y, Sasaki M, Suzuki T, Ogura G and Kumaki N: Salivary duct carcinoma with invasive micropapillary and rhabdoid feature arising in the submandibular gland. Tokai J Exp Clin Med 42: 30-36, 2017.

18. Kitagawa H, Yoshimitsu M, Kaneko M, Ibuki Y, Emi M, Kohashi T, Mukaida $\mathrm{H}$, Matsuura $\mathrm{H}$, Ohge $\mathrm{H}$, Ohdan $\mathrm{H}$, et al: Invasive micropapillary carcinoma component is an independent prognosticator of poorer survival in Stage III colorectal cancer patients. Jpn J Clin Oncol 47: 1129-1134, 2017.

19. Kuroda $\mathrm{N}$ and Yorita K: Colon cancer with micropapillary carcinoma component: A clinopathologic study of 9 cases. Pol J Pathol 68: 102-108, 2017.

20. Cserni G: Reversed polarity of the glandular epithelial cells in micropapillary carcinoma of the large intestine and the EMA/MUC ${ }_{1}$ immunostain. Pathology 46: 527-532, 2014.

21. Alsadoun N, MacGrogan G, Truntzer C, Lacroix-Triki M, Bedgedjian I, Koeb MH, Alam EEl, Medioni D, Parent M, Wuithier P, et al: Solid papillary carcinoma with reverse polarity of the breast harbors specific morphologic, immunohistochemical and molecular profile in comparison with other benign or malignant papillary lesions of the breast: A comparative study of 9 additional cases. Mod Pathol 31: 1367-1380, 2018.

22. Lotan TL, Ye H, Melamed J, Wu XR, Shih IeM and Epstein JI: Immunohistochemical panel to identify the primary site of invasive micropapillary carcinoma. Am J Surg Pathol 33: 1037-1041, 2009.

23. Terada T: An immunohistochemical study of primary signet-ring cell carcinoma of the stomach and colorectum: II. Expression of $\mathrm{MUC}_{1}, \mathrm{MUC}_{2}, \mathrm{MUC}_{5} \mathrm{AC}$, and $\mathrm{MUC}_{6}$ in normal mucosa and in 42 cases. Int J Clin Exp Pathol 6: 613-621, 2013.

24. Park SY, Lee HS, Choe G, Chung JH and Kim WH: Clinicopathological characteristics, microsatellite instability, and expression of mucin core proteins and p53 in colorectal mucinous adenocarcinomas in relation to location. Virchows Arch 449: 40-47, 2006.

25. Duncan TJ, Watson NF, AI-Attar AH, Scholefield JH and Durrant LG: The role of $\mathrm{MUC}_{1}$ and $\mathrm{MUC}_{3}$ in the biology and prognosis of colorectal cancer. World J Surg Oncol 5: 31, 2007.

26. Wang Y, Srinivasan K, Siddiqui MR, George SP, Tomar A and Khurana S: A novel role for villin in intestinal epithelial cell survival and homeostasis. J Biol Chem 283: 9454-9464, 2008.

27. Gonzalez RS, Huh WJ, Cates JM, Washington K, Beauchamp RD, Coffey RJ and Shi C: Micropapillary colorectal carcinoma: Clinical, pathological and molecular properties, including evidence of epithelial-mesenchymal transition. Histopathology 70: 223-231, 2017.

28. Uhlén M, Fagerberg L, Hallström BM, Lindskog C, Oksvold P, Mardinoglu A, Sivertsson Å, Kampf C, Sjöstedt E, Asplund A, et al: Proteomics. Tissue-based map of the human proteome. Science 347: 1260419, 2015.

29. Wilson LA, Heraty L, Ashford BA, Coelho S, Frangi AF, Pozo JM, Ince PG and Highley JR: Tissue microarray (TMA) use in post mortem neuropathology. J Neurosci Methods 347: $108963,2020$.

30. Uhlen M, Zhang C, Lee S, Sjöstedt E, Fagerberg L, Bidkhori G, Benfeitas R, Arif M, Liu Z, Edfors F, et al: A pathology atlas of the human cancer transcriptome. Science 357: eaan2507, 2017.

31. Eom DW, Kang GH, Han SH, Cheon GJ, Han KH, Oh HS, Kim JH, Jang HJ and Hong SM: Gastric micropapillary carcinoma: A distinct subtype with a significantly worse prognosis in TNM stages I and II. Am J Surg Pathol 35: 84-91, 2011.

32. Hattori T, Sentani K, Hattori Y, Oue N and Yasui W: Pure invasive micropapillary carcinoma of the esophagogastric junction with lymph nodes and liver metastasis. Pathol Int 66: $583-586,2016$ 
33. Lee HJ, Eom DW, Kang GH, Han SH, Cheon GJ, Oh HS, Han KH, Ahn HJ, Jang HJ and Han MS: Colorectal micropapillary carcinomas are associated with poor prognosis and enriched in markers of stem cells. Mod Pathol 26: 1123-1131, 2013.

34. Xu F, Xu J, Lou Z, Di M, Wang F, Hu H and Lai M: Micropapillary component in colorectal carcinoma is associated with lymph node metastasis in $\mathrm{T}_{1}$ and $\mathrm{T}_{2}$ stages and decreased survival time in TNM stages I and II. Am J Surg Pathol 33: 1287-1292, 2009.

35. Verdú M, Román R, Calvo M, Rodón N, García B, González M, Vidal A and Puig X: Clinicopathological and molecular characterization of colorectal micropapillary carcinoma. Mod Pathol 24: 729-738, 2011.

36. Miyazaki M, Aoki M, Okado Y, Koga K, Hamasaki M, Kiyomi F, Sakata T, Nakagawa T and Nabeshima K: Poorly differentiated clusters predict a poor prognosis for external auditory canal carcinoma. Head Neck Pathol 13: 198-207, 2019.

37. Barresi V, Branca G, Vitarelli E and Tuccari G: Micropapillary pattern and poorly differentiated clusters represent the same biological phenomenon in colorectal cancer: A proposal for a change in terminology. Am J Clin Pathol 142: 375-383, 2014.

38. Zlobec I and Lugli A: Epithelial mesenchymal transition and tumor budding in aggressive colorectal cancer: Tumor budding as oncotarget. Oncotarget 1: 651-661, 2010.
39. Lino-Silva LS, Salcedo-Hernández RA and Caro-Sánchez CH: Colonic micropapillary carcinoma, a recently recognized subtype associated with histological adverse factors: Clinicopathological analysis of 15 cases. Colorectal Dis 14: e567-e572, 2012.

40. Yang YL, Liu BB, Zhang X and Fu L: Invasive micropapillary carcinoma of the breast: An update. Arch Pathol Lab Med 140: 799-805, 2016

41. Dalle Vedove A, Falchi F, Donini S, Dobric A, Germain S, Di Martino GP, Prosdocimi T, Vettraino C, Torretta A, Cavalli A, et al: Structure-based virtual screening allows the identification of efficient modulators of e-cadherin-mediated cell-cell adhesion. Int J Mol Sci 20: E3404, 2019.

42. Revenu C, Courtois M, Michelot A, Sykes C, Louvard D and Robine S: Villin severing activity enhances actin-based motility in vivo. Mol Biol Cell 18: 827-838, 2007.

(i) (9) This work is licensed under a Creative Commons c) At No ND Atribution-NonCommercial-NoDerivatives 4.0 International (CC BY-NC-ND 4.0) License. 